\title{
Emploi de microsatellites pour l'analyse de la diversité génétique des races bovines françaises : premiers résultats
}

\author{
K Moazami-Goudarzi, D Vaiman, D Mercier, C Grohs, \\ JP Furet, H Levéziel, P Martin \\ Institut national de la recherche agronomique, \\ laboratoire de génétique biochimique et cytogénétique, \\ 78352, Jouy-en-Josas Cedex, France
}

\begin{abstract}
Résumé - La caractérisation des races bovines françaises a débuté en utilisant comme principales sources de données des études morphologiques. Progressivement, grâce à l'évolution des techniques d'analyse de la variabilité génétique, d'autres critères ont pu être pris en compte. Des données concernant le polymorphisme des groupes sanguins, de certaines protéines sériques et des protéines du lait ont permis de distinguer 4 sousensembles de races bovines françaises. Cette étude va être approfondie par une analyse du polymorphisme au niveau de l'ADN en utilisant de nouveaux marqueurs plus nombreux et plus polymorphes : les microsatellites. Cet article présente la mise en place d'un projet d'étude du polymorphisme de 20 microsatellites dans 11 races bovines françaises choisies de façon à ce que les 4 sous-ensembles soient représentés. Les premiers résultats obtenus sur 6 races (Brune des Alpes (BU), Charolaise ( $\mathrm{CH})$, Montbéliarde (MO), Normande (NO), Parthenaise (PA) et Vosgienne (VO) ) avec six locus microsatellites (INRA013, INRA016, INRA035, INRA037, INRA040 et ETH131) sont décrits. Une répartition très différente des fréquences alléliques des microsatellites est observée d'une population à l'autre. Ces résultats préliminaires montrent que les microsatellites peuvent être de bons outils pour ce programme d'analyse phylogénique.
\end{abstract}

bovin / polymorphisme / race / microsatellite

Summary - Analysis of genetic diversity in French cattle breeds by the use of microsatellites: preliminary results. French cattle breeds were first characterized according to data from morphological studies. Subsequently, technical advances allowed the use of other polymorphic biochemical characters such as blood groups, serum proteins or milk proteins. The different breeds could be classified into 4 subsets. At present, the development of molecular biology techniques has made it possible to analyse polymorphism at the DNA level using microsatellites which are numerous and highly polymorphic. This article presents a study on the polymorphism of 20 microsatellites in 11 French cattle breeds including representatives of the 4 subsets. The first results obtained with 6 cattle breeds (Brune des Alpes (BU), Charolaise ( $\mathrm{CH}$ ), Montbéliarde (MO), Normande (NO), Parthenaise (PA) and Vosgienne (VO)) using 6 microsatellite loci (INRA013, INRA016, 
INRA035, INRA037, INRA040 and ETH131) are described. Differences were observed in microsatellite allelic frequencies between the different breeds. These prelimary results show that microsatellites are promising tools for phylogenetic studies.

bovine / polymorphism / breed / microsatellite

\section{INTRODUCTION}

La variabilité génétique des populations bovines françaises est indispensable à l'amélioration des productions animales. Or, depuis quelques dizaines d'années, la sélection pour un nombre limité de caractères (production laitière, croissance) s'est intensifiée. Elle a été menée de manière efficace selon les méthodes de la génétique quantitative et a été accompagnée de la standardisation des systèmes d'élevage. Aussi, de nombreuses races ont vu leurs effectifs diminuer dramatiquement au bénéfice de celles répondant le mieux aux enjeux économiques ( 5 races constituent $90 \%$ du cheptel actuel). Dans ce contexte, la protection et la gestion de la diversité génétique, en vue de l'exploitation et de la conservation du patrimoine existant, apparaissent de plus en plus nécessaires.

Les outils et méthodes utilisés pour appréhender ou contrôler cette diversité étaient jusqu'à présent surtout basés sur l'observation des caractères visibles (Lauvergne, 1989), sur l'analyse des données démographiques et généalogiques dans les populations (voir revue par Vu Tien Khang, 1983) ou sur l'examen de l'évolution des variances génétiques dans les populations soumises à sélection (Verrier et al, 1989). Les apports de la génétique biochimique ont offert de nouvelles perspectives (Grosclaude, 1974), grâce à la description des polymorphismes génétiques révélés par des méthodes immunologiques (groupes sanguins) ou électrophorétiques (protéines du lait, protéines sériques ou érythrocytaires). Le processus de sélection intensif étant engagé avant le développement de ces possibilités d'études du génome (Vissac, 1978), celles-ci n'ont pour l'instant que peu influencé la sélection. Elles ont cependant fourni des moyens appropriés pour l'identification des animaux et le contrôle de filiation.

Une autre utilisation des polymorphismes biochimiques réside dans l'analyse des relations génétiques entre races, afin d'apprécier leur degré d'originalité et d'orienter les programmes de conservation (Guérin, 1980). Aussi, des travaux visant à évaluer les distances génétiques entre races ont été développés. En France, une seule étude menée par Grosclaude et al (1990), s'appuyant sur l'analyse de 13 systèmes génétiques indépendants ( 11 groupes sanguins, transferrine sérique et caséine $\beta$ ), a récemment conduit à distinguer, parmi 18 races bovines françaises, 4 sous-ensembles de races. Une des conclusions de cette étude était qu'une analyse plus approfondie, effectuée au moyen de systèmes génétiques polymorphes plus nombreux et bien répartis dans l'ensemble du génome, devrait permettre d'appréhender plus finement les différences entre races.

Même en considérant les travaux sur le complexe majeur d'histocompatibilité (complexe BoLA), le nombre de marqueurs génétiques, décrits par des travaux portant sur les produits des gènes, est resté limité (Larsen et al, 1992). Le 
développement des méthodes de biologie moléculaire a depuis quelques années profondément modifié et amélioré le pouvoir d'investigation des chercheurs, puisque l'ensemble du polymorphisme de l'ADN est maintenant accessible.

Aujourd'hui, dans le cadre du développement des travaux sur la cartographie génétique des bovins (Gellin et Levéziel, 1992), l'augmentation très rapide du nombre de marqueurs génétiques de type microsatellite décrits, a offert la possibilité de compléter les travaux antérieurs avec de nouveaux outils. Les microsatellites sont des séquences nucléotidiques constituées de répétitions en tandem de motifs simples (par exemple (TG) $n$ avec $10<n<30$ ), le nombre de répétitions du motif variant d'un allèle à l'autre. Dans toutes les espèces de mammifères étudiées, leur nombre est estimé entre 50 et 100000 loci; de plus, un polymorphisme très important a été rapporté et leur bonne répartition dans le génome mis en évidence (Stalling et al, 1991).

Ces diverses caractéristiques font considérer les microsatellites comme des marqueurs de choix susceptibles de fournir une meilleure appréciation et une caractérisation plus fine de la diversité génétique des races. Nous avons donc entrepris une étude se limitant dans un premier temps à l'analyse du polymorphisme de 20 microsatellites dans 11 races bovines françaises. Sa mise en place ainsi que les premiers résultats sont reportés et les perspectives discutées.

\section{MATÉRIELS ET MÉTHODES}

\section{Animaux}

Compte tenu de l'importance des travaux de laboratoire à réaliser, l'étude entreprise a été limitée à l'examen de 11 races bovines françaises. Dix d'entre elles ont été choisies en fonction des travaux antérieurs de Grosclaude et al (1990), afin que chacun des 4 sous-ensembles de races identifiés alors soit représenté. Ainsi ont été échantillonnées : 1) dans le groupe des races du Nord : Maine-Anjou $(\mathrm{MA})$; 2) dans le groupe des races du Centre et du Sud-Ouest : Charolaise (CH), Limousine (LI); 3) dans le groupe des races de l'Ouest et de l'Est : Bretonne Pie-Noire (BR), Parthenaise (PA), Brune des Alpes (BU), Montbéliarde (MO), Vosgienne (VO); 4) et la Normande (NO) constituant à elle seule un groupe. La race Hosltein $(\mathrm{HO})$ et la Jersiaise $(\mathrm{JE})$ ont de plus été prises en compte en temps que témoins internationaux. Dans chacune des races, un échantillon d'une cinquantaine d'animaux non apparentés (ou le moins possible d'après l'examen des généalogies sur deux générations) a été constitué. Il s'agit de femelles, sauf pour la race Limousine, appartenant soit à des élevages expérimentaux de l'INRA, soit à des élevages privés.

\section{Extraction d'ADN génomique}

Pour chaque animal, l'extraction de l'ADN génomique a été effectuée selon le protocole de Jeanpierre (1987), à partir de $25 \mathrm{ml}$ de sang total prélevé sur EDTA tripotassique. Après addition de $30 \mathrm{ml}$ de $\mathrm{NE}(\mathrm{NaCl} 10 \mathrm{mM}, \mathrm{EDTA} 10 \mathrm{mM}, \mathrm{pH} 7)$, les tubes sont mis sous agitation pendant $1 \mathrm{~h}$ à $4^{\circ} \mathrm{C}$ puis centrifugés $20 \mathrm{~min}$ à $2000 \mathrm{rpm}$. Les culots leucocytaires sont repris dans $50 \mathrm{ml}$ de $\mathrm{NE}$ et lavés à 3 reprises 
dans les mêmes conditions, le dernier lavage étant écourté à $15 \mathrm{~min}$. Les culots sont resuspendus et incubés une nuit à $58^{\circ} \mathrm{C}$ dans $14 \mathrm{ml}$ d'une solution de chlorure de guanidine $6 \mathrm{M}, 1 \mathrm{ml}$ d'acétate d'ammonium $7,5 \mathrm{M}, 1 \mathrm{ml}$ de N-Lauroylsarcosine $20 \%$, additionnée de $4 \mathrm{mg}$ de protéinase $\mathrm{K}$ (Boehringer Mannheim GmbH). L'ADN génomique est ensuite obtenu par précipitation en présence de $30 \mathrm{ml}$ d'éthanol absolu. Après plusieurs lavages à l'éthanol $70 \%$ et dissolution totale dans une solution de Tris-HCl $10 \mathrm{mM}$, EDTA $1 \mathrm{mM}$ (TE 10,1), les concentrations d'ADN sont ajustées à $200 \mu \mathrm{g} / \mathrm{ml}$.

\section{Microsatellites}

Les microsatellites utilisés dans cette étude ont été sélectionnés selon leur disponibilité parmi ceux récemment produits au laboratoire ou publiés. Le choix a été effectué en fonction, d'une part, de leurs caractéristiques techniques (bonne aptitude à l'amplification et interprétation aisée des typages), et d'autre part, de leurs caractérisques génétiques (degré de polymorphisme $=$ nombre d'allèles, localisation et répartition dans le génome). Une vingtaine de marqueurs génétiques ont ainsi été retenus (tableau I). Nous présentons les premiers résultats obtenus, dans l'analyse des races Brune des Alpes (BU), Charolaise ( $\mathrm{CH}$ ), Montbéliarde (MO), Normande (NO), Parthenaise (PA) et Vosgienne (VO), avec 6 locus microsatellites : INRA013, INRA016, INRA035, INRA037, INRA040 et ETH131.

Tableau I. Caractéristiques principales des microsatellites sélectionnés.

\begin{tabular}{|c|c|c|c|c|}
\hline Microsatellites & $\begin{array}{l}\text { Nombres } \\
\text { d'allèles }\end{array}$ & $\begin{array}{l}\text { Groupe de } \\
\text { synténie : } U\end{array}$ & $\begin{array}{c}\text { Chromosome: } \\
\text { Chr }\end{array}$ & $\begin{array}{c}\text { Références } \\
\text { bibliographiques }\end{array}$ \\
\hline INRAK & 6 & U15 & Chr6 & Levéziel et al, 1994 \\
\hline INRA05 & 3 & U27 & & Vaiman et al, 1992 \\
\hline INRA011 & 8 & U10 & Chr1 & Vaiman et al, 1992 \\
\hline INRA013 & 11 & U1 & Chr16 & Vaiman et al, 1992 \\
\hline INRA016 & 10 & non assigné & & Vaiman et al, 1993 \\
\hline INRA023 & 12 & $\mathrm{U} 6$ & Chr3 & Vaiman et al, 1993 \\
\hline INRA025 & 16 & $\mathrm{U} 23$ & Chr17 & Vaiman et al, 1993 \\
\hline INRA032 & 6 & U16 & Chr11 & Vaiman et al, 1993 \\
\hline INRA035 & 10 & U1 & Chr16 & Vaiman et al, 1993 \\
\hline INRA037 & 11 & U5 & Chr10 & Vaiman et al, 1993 \\
\hline INRA040 & 43 & U17 & Chr2 & Vaiman et al, 1993 \\
\hline INRA063 & 7 & U9 & Chr18 & Vaiman et al, 1993 \\
\hline INRA064 & 5 & U20 & Chr23 & Vaiman et al, 1993 \\
\hline INRA072 & 11 & U13 & $\mathrm{Chr} 4$ & Vaiman et al, 1993 \\
\hline ETH131 & 14 & $\mathrm{U} 4$ & Chr21 & Steffen et al, 1993 \\
\hline ETH152 & 9 & U2 & Chr9 & Steffen et al, 1993 \\
\hline ETH225 & 6 & U3 & Chr5 & Steffen et al, 1993 \\
\hline
\end{tabular}




\section{Typage des animaux}

\section{PCR radioactive}

L'amplification est effectuée dans un volume réactionnel de $10 \mu \mathrm{l}$ contenant $200 \mathrm{ng}$ d'ADN génomique, $25 \mu \mathrm{M}$ des 3 désoxynucléotides dCTP, dGTP et dTTP, 2,5 $\mu \mathrm{M}$ de dATP, $1 \mu \mathrm{Ci}$ de $\left[\mathrm{S}^{35}\right.$-dATP, $1,5 \mu \mathrm{M}$ de $\mathrm{MgCl}_{2}, 0,5$ unités de Taq polymérase (Promega), $20 \mathrm{pM}$ de chaque amorce, dans un tampon Tris- $\mathrm{HCl} 10 \mathrm{mM}$ (pH 9), $\mathrm{KCl} 50 \mathrm{mmol}$. Chaque cycle d'amplification comporte 3 étapes : 1) $30 \mathrm{~s}$ à $94^{\circ} \mathrm{C}$, 2) $30 \mathrm{~s}$ à $\left.55-65^{\circ} \mathrm{C}, 3\right) 30 \mathrm{~s}$ à $72^{\circ} \mathrm{C}$. La réaction de $\mathrm{PCR}$ est réalisée à l'aide d'un appareil Perkin-Elmer Cetus 9600 et comprend une dénaturation initiale de $5 \mathrm{~min}$ à $94^{\circ} \mathrm{C}, 30$ cycles d'amplification et une élongation finale de $5 \min$ à $72^{\circ} \mathrm{C}$.

\section{Electrophorèse}

L'amplifiat est dénaturé $4 \mathrm{~min}$ à $80^{\circ} \mathrm{C}$ et $7,5 \mu \mathrm{l}$ sont déposés sur un gel de polyacrylamide-urée $5 \%$. La migration électrophorétique est effectuée à 1500-1600 volts constants, sa durée étant variable selon la taille de l'amplifiat (de 1,5 à $3 \mathrm{~h}$ ). Le gel est ensuite transféré sur papier Whatmann $3 \mathrm{MM}$, puis mis à șécher pendant $1 \mathrm{~h}$ à $80^{\circ} \mathrm{C}$, avant autoradiographie. La durée d'exposition du film peut varier de un jour à une semaine suivant l'intensité du signal.

\section{Détermination des génotypes et nomenclature}

Les génotypes sont déterminés par lecture des autoradiographies, en s'aidant d'échantillons témoins et d'une séquence M13, déposés sur les gels. La désignation des allèles de 1 à $n$, pour chaque microsatellite, correspond au niveau et à l'ordre de migration des amplifiats : 1 et $n$ désignent respectivement ceux de plus grande et de plus petite tailles observées. La numérotation des allèles correspond ainsi à des positions alléliques qui différent les unes des autres par 2 bases. Il faudra donc distinguer le nombre d'allèles théoriques $(n)$ du nombre d'allèles réellement observé.

\section{Calculs}

Les fréquences alléliques sont calculées par comptage direct et, pour chaque locus, la valeur de PIC (polymorphism information content) est obtenue selon Botstein et al (1980).

\section{RÉSULTATS}

Ces résultats ne décrivent qu'une partie préliminaire du projet de recherche développé au laboratoire.

La figure 1 montre la répartition des fréquences alléliques du microsatellite INRA016 par race. La plupart des allèles sont trouvés dans toutes les races, mais une nette variabilité interraciale est observée. En effet, l'allèle 5 est rencontré à une fréquence de 0,32 chez les Charolais et de 0,07 en Brune des Alpes. Il en est de même pour l'allèle 7 , lorsque l'on compare les Montbéliardes $(0,29)$ et les Brune des 
Alpes $(0,04)$. Deux situations extrêmes sont à noter : dans plusieurs races, certains allèles sont absents (par exemple, l'allèle 6 n'a pas été détecté en Parthenaise, ni l'allèle 4 en Brune des Alpes) alors que l'allèle -1 n'est présent qu'en Normande. La notation -1 a dû être introduite du fait de la présence d'un allèle correspondant à un fragment d'amplification de taille supérieure à ceux jusqu'alors détectés.

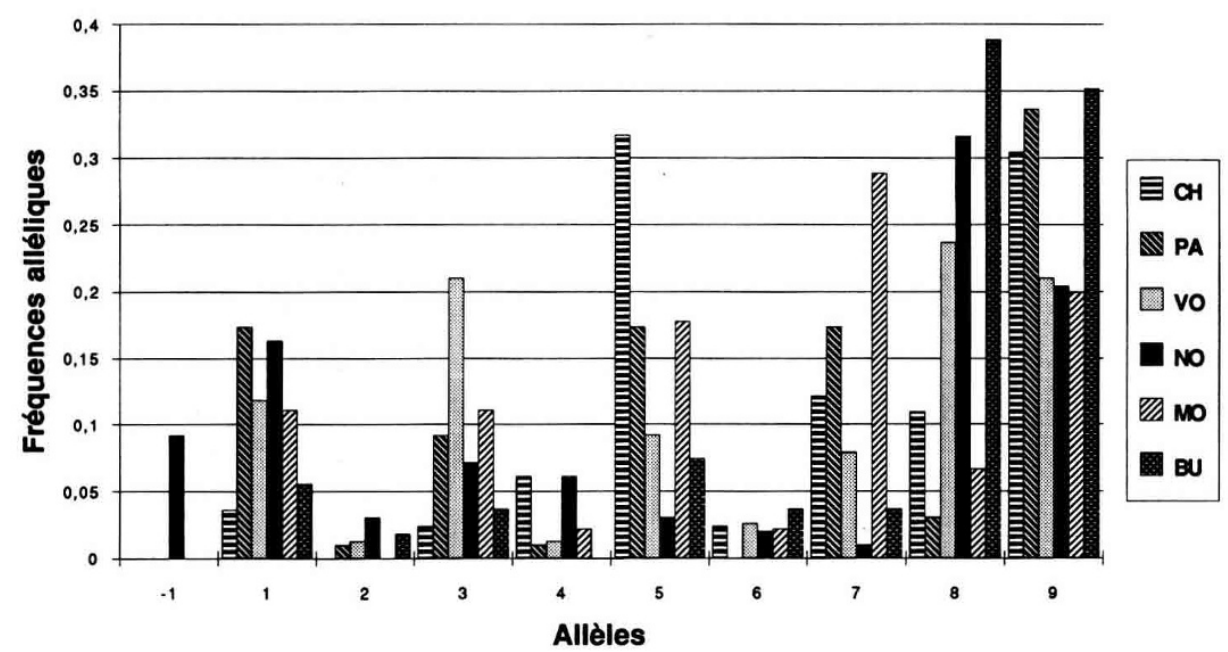

Fig 1. Fréquences alléliques par race du microsatellite INRA016. Code races : voir Matériels et méthodes.

La figure 2 montre la répartition des fréquences alléliques des allèles du microsatellite INRA035 dans les 6 races analysées. Alors que le nombre d'allèles observés pour ce marqueur est similaire à celui relaté pour INRA016, le profil de répartition est remarquablement différent. En effet, 2 allèles (8 et 9) sont prépondérants dans chacune des races.

La figure 3 illustre cette observation : sur les 14 animaux Montbéliard présentés, l'allèle 4 n'est rencontré qu'une fois alors que les allèles 8 ou 9 sont détectés chez tous les autres animaux. Seul l'allèle 10 a été observé chez les Charolais, cette situation rappelant celle décrite dans le cas de l'allèle -1 du microsatellite INRA016 détecté en race Normande.

Les valeurs de PIC calculées pour les 6 microsatellites sont indiquées, par race, dans le tableau II. La localisation ainsi que les nombres d'allèles théoriques et observés sont également mentionnés. D'une manière générale, les valeurs de PIC sont assez élevées. Les plus grandes sont obtenues pour le microsatellite INRA040, en accord avec le nombre important d'allèles. Sur les 6 microsatellites étudiés, 5 d'entre eux ont des valeurs moyennes de PIC qui sont comparables à celle que l'on pourrait calculer en supposant une répartition homogène des allèles. Seul le microsatellite INRA035, a une valeur moyenne de PIC $(0,5)$ inférieure à la valeur théorique $(0,89)$ du fait de la mauvaise distribution des allèles notamment chez les Normand et Montbéliard. Par ailleurs, la moyenne des valeurs de PIC obtenues en Charolaise $(0,74)$ pourrait suggérer globalement un polymorphisme plus important 


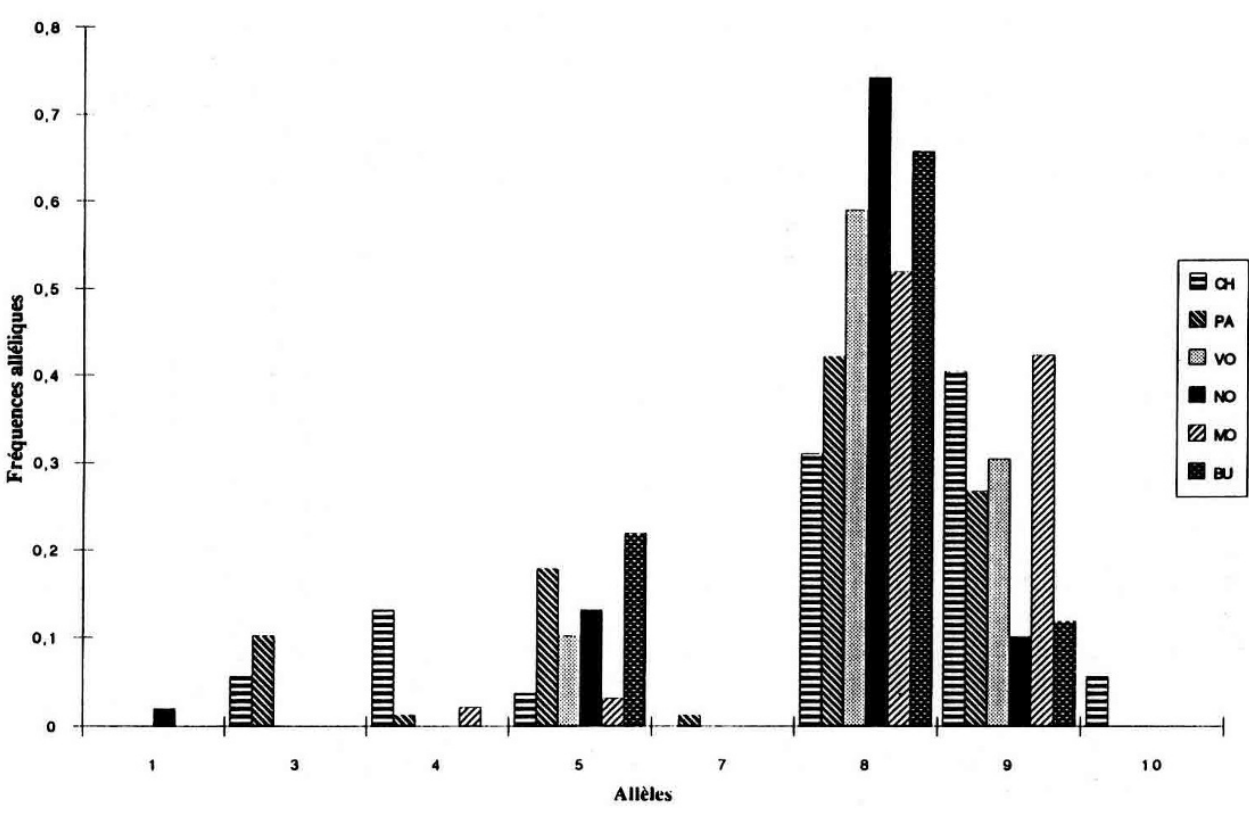

Fig 2. Fréquences alléliques par race du microsatellite INRA016. Code races : voir Matériels et méthodes.

dans cette race. La valeur la plus faible se rencontre en Brune des Alpes $(0,66)$, les autres races étant en situation intermédiaire (environ 0,70 ).

\section{DISCUSSION}

L'étude entreprise sur 11 races en utilisant 20 microsatellites s'est mise en place progressivement. La constitution des échantillons raciaux a représenté un travail certain (choix des animaux, prélèvements, extractions d'ADN), et a été répartie sur plusieurs hivers. La taille des échantillons a été fixée à 50 individus non apparentés, pour permettre a priori une bonne couverture de la diversité génétique des races étudiées sans trop alourdir le dispositif expérimental. Cette taille d'échantillon est habituellement acceptée comme conforme pour ce type d'étude (Nei et Roychoudhury, 1974; Nei, 1978), mais il ne faut pas négliger les risques existants. En effet, à taille égale, il est évident que la représentativité n'est pas la même en fonction des effectifs des races. De plus, dans le cas des systèmes extrêmement polymorphes comme INRA040, il est clair que l'estimation des fréquences alléliques est moins précise. Il conviendra donc d'être prudent quand aux remarques à faire lors de l'observation d'allèles rares.

Le choix des microsatellites a en fait été conditionné par leur disponibilité. Au départ, quelques-uns parmi les premiers produits au laboratoire ont été retenus sans que l'information concernant leur localisation soit disponible. En effet, tout nouveau microsatellite isolé a été systématiquement caractérisé pour son polymorphisme 


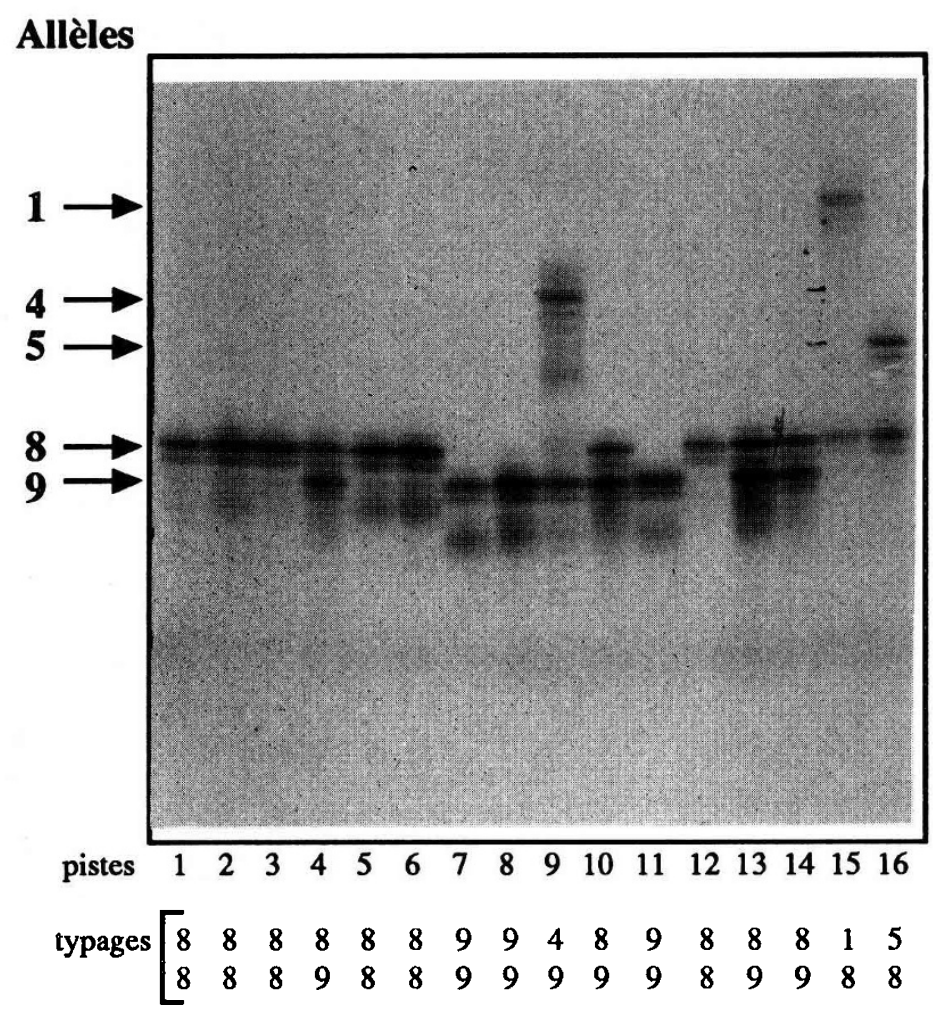

Fig 3. Analyse du polymorphisme du microsatellite INRA035 par électrophorèse en gel de polyacrylamide urée en race Montbéliarde. Pistes 1 à 14 : échantillons de race Montbéliarde; pistes 15 et 16 : témoins de race Normande. Les génotypes des animaux sont indiqués ainsi : sur la piste 1 , l'animal est homozygote pour l'allèle 8 , lire $8 / 8$.

Tableau II. Calcul des valeurs de PIC (polymorphism information content).

\begin{tabular}{lccccccccccc}
\hline $\begin{array}{l}\text { Micro- } \\
\text { satellites }\end{array}$ & Localisation & $\begin{array}{c}\text { Nb allèles } \\
\text { théoriques }\end{array}$ & $\begin{array}{c}\text { Nb allèles } \\
\text { observés }\end{array}$ & $C H$ & $P A$ & $V O$ & $N O$ & $M O$ & $B U$ & Moyenne \\
\hline INRA013 & U1 chr16 & 11 & 10 & 0,78 & 0,74 & 0,77 & 0,71 & 0,67 & 0,60 & 0,71 \\
INRA016 & non assigné & 10 & 10 & 0,74 & 0,62 & 0,80 & 0,79 & 0,79 & 0,67 & 0,74 \\
INRA035 & U1 chr16 & 10 & 8 & 0,67 & 0,66 & 0,47 & 0,38 & 0,37 & 0,45 & 0,50 \\
INRA037 & U5 chr10 & 15 & 15 & 0,82 & 0,61 & 0,68 & 0,63 & 0,71 & 0,59 & 0,67 \\
INRA040 & U17 chr2 & 44 & 37 & 0,69 & 0,75 & 0,85 & 0,91 & 0,90 & 0,93 & 0,84 \\
ETH131 & U4 chr21 & 14 & 13 & 0,75 & 0,81 & 0,64 & 0,81 & 0,74 & 0,69 & 0,74 \\
\hline
\end{tabular}

Pour la localisation : $\mathrm{U}=$ groupe de synténie, $\mathrm{chr}=$ chromosome. 
sur un panel de 40 animaux appartenant à 8 races différentes. Par la suite, grâce aux méthodes de cartographie physique ou génétique, ces marqueurs ont été localisés plus finement et sélectionnés de manière plus aisée. Cela explique pourquoi INRA013 et INRA035 appartiennent au même groupe de synténie et l'estimation de la distance entre eux apportera un complément d'information pertinent. L'utilisation de ETH131 est justifiée par le fait qu'il était le seul appartenant à U4 (Chr21).

La désignation des allèles est établie selon les observations initiales réalisées sur notre panel de 40 animaux. Elle a dû être adaptée au cours de l'étude d'un nombre croissant d'animaux, du fait de l'existence d'allèles nouveaux. Il s'agit d'une difficulté pratique importante sans solution parfaite, ce qui explique l'absence actuelle de régles internationales.

Les résultats obtenus confirment l'existence d'un polymorphisme important et surtout d'une grande diversité de situations, soit entre marqueurs, soit entre races. La distribution des allèles révèle très finement la variabilité génétique présente dans les différentes races.

Le calcul des valeurs de PIC reflète le polymorphisme détecté. Cet indice synthétique est souvent calculé sur un nombre limité d'animaux afin d'obtenir une estimation de la qualité d'un marqueur pour des études génétiques (étude de ségrégation, identification et contrôle de paternité, étude de populations). Les données recueillies montrent néanmoins que cette information n'est pas seule suffisante pour juger de la bonne opportunité d'utiliser un marqueur plutôt qu'un autre selon les travaux envisagés. En effet, à nombre égal d'allèles observés, des PIC différents sont évalués dans les diverses races. De plus, à valeur de PIC égale, les distributions d'allèles sont très variables. L'examen d'échantillons raciaux de taille suffisante, pour chaque microsatellite, apparaît donc nécessaire.

L'étude entreprise vise ainsi à acquérir un ensemble important d'information sur les races bovines françaises, afin de décrire plus finement la variabilité génétique existante. L'analyse des données sera effectuée par le calcul des distances génétiques et permettra de compléter les travaux antérieurs. Les nouvelles informations obtenues devraient offrir la possibilité d'appréhender les situations décrites comme particulières par Grosclaude et al (1990), à savoir le rattachement de la race Charolaise au même groupe que les races blondes du Sud-Ouest ou le rattachement de la Parthenaise et de la Bretonne Pie Noire au groupe des races de l'Est.

Mais la difficulté majeure reste le fait qu'il faut accumuler une grande quantité d'information. Le développement de ces travaux est largement dépendant des progrès d'automatisation des analyses et de la diminution des coûts. L'emploi de séquenceurs automatiques, et d'amorces fluorescentes, offre des perspectives réelles. Des efforts importants sont aujourd'hui déployés pour rendre effective la détermination simultanée du génotype d'un animal à 8 loci. De plus, ces automates permettent une acquisition rapide et sûre des données. En définitive, nos premiers résultats, obtenus dans l'étude de 6 races à l'aide de 6 locus microsatellites, confirment qu'ils sont des marqueurs intéressants pour l'étude de la diversité génétique des races dans la mesure ou une très forte variabilité interraciale est révélée. La poursuite de notre travail favorisera l'introduction de l'analyse génétique au niveau moléculaire dans la description des populations. Cela aura pour conséquence de fournir des indices objectifs, nécessaires à une meilleure analyse de la diversité génétique 
en vue de sa gestion et de son exploitation. Il sera ainsi envisageable de développer des investigations dans un nombre plus important de races afin de préciser le réel degré d'originalité de certaines d'entre elles sur lesquelles pourraient se concentrer les efforts de conservation.

\section{REMERCIEMENTS}

Les auteurs tiennent à remercier l'ensemble des personnes et des organismes professionnels ayant aidé à la collecte des échantillons raciaux. Ils sont également redevables à F. Grosclaude d'un soutien constant et précieux dans le développement de ce travail. Cette recherche a bénéficié du soutien du programme Agrotech de 1'INRA (Projet Prodige).

\section{RÉFÉRENCES}

Botstein D, White RL, Skolnick M, Davis RW (1980) Construction of a genetic linkage map in man using restriction fragment length polymorphisms. Am J Hum Genet 32, 314-331

Gellin J, Levéziel H (1992) Apports actuels et futurs des marqueurs génétiques dans l'amélioration des populations animales. Stratégie d'établissement des cartes géniques des espèces d'élevage. In: INRA Prod Anim (numéro hors série), 281-286 Grosclaude F (1974) Exposé d'introduction, Séance plénière Génétique biochimique. In: Ier Congrès mondial de génétique appliquée à l'élevage. Editorial Garsi Madrid, Octobre 1974, vol 1, 229-241

Grosclaude F, Aupetit RY, Lefebvre J, Mériaux JC (1990) Essai d'analyse des relations génétiques entre les races bovines françaises à l'aide du polymorphisme biochimique. Genet Sel Evol 22, 317-338

Guérin G (1980) Le polymorphisme biochimique et l'analyse des relations génétiques entre races. Bull Tech Inf 351-352, 437-446

Jeanpierre M (1987) A rapid method for the purification of DNA from blood. Nucleic Acids Res 15, 9611

Larsen B, Di Stasio L, Tucker EM (1992) List of alleles for blood and milk proteins in cattle, sheep and goat. Anim Genet 23, 188-192

Lauvergne JJ (1989) La constitution des ressources génétiques animale de ferme. In: La gestion des ressources génétiques des espèces animales domestiques (M Molénat et $E$ Verrier, eds). BRG, Paris, 9-18

Levéziel H, Rodellar C, Leroux C et al (1994) A microsatellite within the bovine $\mathrm{k}$-casein gene reveals a polymorphism correlating strongly with polymorphisms previously described at the protein as well as at the DNA level. Anim Genet (sous presse)

Nei M, Roychoudhury AK (1974) Sampling variances of heterozygosity and genetic distance. Genetics 76, 379-390

Nei M (1978) Estimation of average heterozygosity and genetic distance from a small number of individuals. Genetics $89,583-590$

Stalling AF, Nelson D, Torney DC, Hildebrand CE, Moyzis RK (1991) Evolution and distribution of (GT)n repetitive sequences in mammalian genomes. Genomics $10,807-815$ 
Steffen P, Eggen A, Dietz A, Womack J, Stranzinger G, Fries R (1993) Isolation and characterization of polymorphic microsatellites in cattle. Anim Genet 24, 121-124 Vaiman D, Osta R, Mercier D, Grohs C, Levéziel H (1992) Characterization of five new bovine dinucleotide repeats. Anim Genet 23, 537-541

Vaiman D, Mercier D, Moazami-Goudarzi K et al (1994) A set of 99 cattle microsatellites: characterization, synteny mapping and polymorphism. Mamm Gen (sous presse)

Verrier E, Colleau JJ, Foulley JL (1989) Perspectives d'évolution de la variabilité génétique et possibilités de progrès à long terme dans les populations animales sélectionnées. In: La gestion des ressources génétiques des espèces animales domestiques (M Molénat, E Verrier, eds). BRG, Paris, 61-70

Vissac B (1978) L'animal et les progrès de la génétique. Evolution des races d'élevage. Cah Méd Vét 47, 111-120

Vu Tien Khang J (1983) Méthodes d'analyse des données démographiques et généalogiques dans les populations d'animaux domestiques. Genet Sel Evol 15, 263298 